\title{
Structure and Elastic Modulii Of Silicon Nanotubes
}

\author{
Veena Verma $^{1, a}$, K. Dharamvir ${ }^{1, b}$ and V. K. Jindal ${ }^{1, c}$ \\ ${ }^{1}$ Department of Physics, Panjab University, Chandigarh (India). \\ a veenangl@yahoo.co.in \\ beya_dv@yahoo.co.in \\ cjindal@pu.ac.in
}

received paper 14.11.2007, received revised paper 28.12.207, accepted date, 02.01.2008

Keywords: Silicon Nanotubes, Tersoff Potential, Young's Modulus, Poisson Ratio, Shear modulus, square lattice, sp3 hybridization.

\begin{abstract}
Based on the assumption that sp3 hybridization is more stable in bulk silicon, this study is a step forward in understanding the structures and mechanical properties of silicon nanotubes (SiNT). Using the well tested form of Tersoff potential we have calculated cohesive energy and other parameters for SiNT of various diameters and chiralities. Using this potential, the results obtained for bulk silicon are satisfactory, so we expect that the same potential would work well with SiNT as well. We calculated Young' modulus and shear modulus for SiNT. Young's modulus lies in the range of 100- $200 \mathrm{GPa}$ which is about 10-20 times lower than CNT and shear modulus lies between 200-300 GPa. This work shall motivate further theoretical and experimental work in the field of nanostructures.
\end{abstract}

\section{Introduction}

The structure of Silicon nanotubes (SiNTs) is still an open question of fundamental physical and chemical importance, which clearly requires concerted efforts Even though both silicon and carbon are isovalent, their behavior in forming chemical bonds is quite different. For example, the sp2 hybridization is more stable in carbon, whereas the sp3 hybridization is more stable in silicon. Therefore, carbon easily forms graphite, fullerene, and nanotube composed of only sp2 bonds, whereas silicon has been well-known in the form of only diamond structure. Silicon, when reduced to the nanoscale (less than one thousand millionth of a metre) becomes biodegradable and dissolves into the body as silicic acid. The acid is harmless and is the natural form of silicon, usually absorbed from food [1]. Xiao Cheng Zeng, Jaeil Bai and their colleagues [2] at the University of NebraskaLincoln, working with Hideki Tanaka of Okayama University, in Japan, have used the supercomputer, Prairiefire, to model single-walled silicon nanotubes in hexagonal, pentagonal, and square configurations Silicon nanotubes similar to multi walled structures are anticipated . Models of silicon nanotubes created by Xiao Cheng Zeng and his research team appear to behave like a metal, losing the semiconducting properties that have made three-dimensional silicon one of the foundation materials for the modern electronics industry.
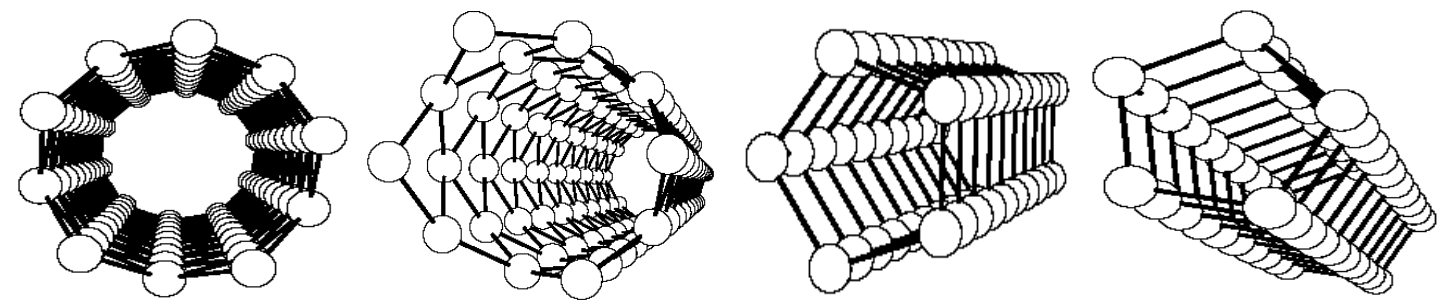

Fig I: $(5,5)$ square zigzag and pentagonal square lattice SiNTs 
The possibility of the existence of silicon nanotubes, had been previously discussed theoretically by several authors, based on density functional theory (DFT), Møller-Plesset electron correlation (MP2) and semi-empirical quantum chemical calculations, as well as molecular dynamics simulations [3-7]. As outlined above, this type of aggregation is particularly surprising for silicon, since $\mathrm{p}$ bonding (and the associated planar sp2-type coordination) is usually necessary to form stable two-dimensional structures (like graphene sheets), tubular structures In fact, with respect to one-dimensional nanostructures, sp3 hybridization favors the formation of Si nanowires [8]. The theoretical models included tubular structures built of hexagons of Si. More recently, a quite different model based on tetragons of sp3-hybridized silicon atoms has been proposed (Fig.2) $[6,7]$.DFT calculations have also been used to predict the mechanical and electronic properties of SiNTs. By calculating sp2 SiNTs, Fagan et al. concluded that the bandgap depends on the chirality, with armchair tubes being metallic, while the chiral tubes are low-bandgap semiconductors. [3] Also, a zero band gap was suggested for sp3 tetragonal SiNTs by Bai et al. [2] Only recently, however, has the synthesis of SiNTs been demonstrated by various groups, each using a different growth process. Yang et al. [10,11] first reported the synthesis by chemical vapor deposition and experimental observation by transmission electron microscopy (TEM) of large-diameter SiNTs Lee and co-workers [5] later reported the growth of SiNTs by molecular beam epitaxy on porous alumina, without using catalysts. More recently, Tang et al. [12,13] demonstrated the self-organized growth of smaller-diameter $(13 \mathrm{~nm})$ SiNTs via hydrothermal synthesis, using silicon monoxide ( $\mathrm{SiO})$ as the starting We are presenting here computed values of the energy per atom, and Young's modulus of SiNTs of different diameter and chirality (both $\mathrm{sp} 2$ and $\mathrm{sp} 3$ bonding) using the well tested form of Tersoff Potential previously used for carbon nanotubes (CNTs) and boron nitride nanotubes (BNNTs) $[14,15]$.

\section{Model And Calculations}

\subsection{Structure}

Taking a cue from the the models of SiNTs [2] and out of curiosity we generated the coordinates of silicon atoms in three types of lattices as shown in fig (2a,2b and 3).Fig.2a represents two dimentional square sheet of silicon in sp3 bonding with Si-Si nearest neighbour distance equal to $2.305 \mathrm{~A}^{\mathrm{O}}$ [16] and when we roll such a square sheet it shall result in forming tetra, penta, hexa and so on depending on the no of silicon atoms on the circumference and we shall call so generated $\mathrm{SiNT}$ as square SiNT. Fig $2 \mathrm{~b}$ represents a triangular lattice of silicon atoms and the distance between silicon atoms on the circumference of SiNT is $3.260 \AA$ and we call this type of SiNT as square zigzag SiNT due to its structure at the end rings. Fig.3 is a well known graphitic sheet of silicon, the rolling of such a sheet can result in zigzag or armchair SiNT. When 2-d lattice of silicon is folded as shown below it forms SiNT of a particular diameter depending on the no of atoms on the circumference and the chirality of the SiNT.

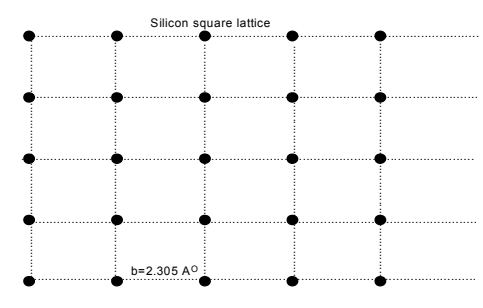

$2 \mathbf{a}$

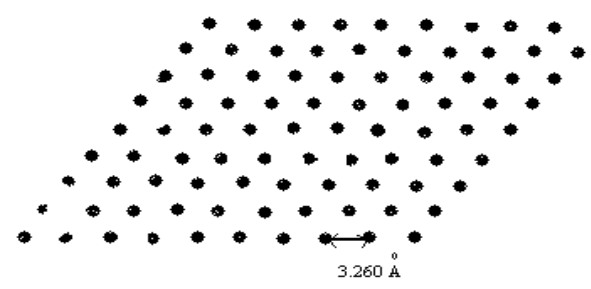

2b

Fig 2:Two dimensional (2a) square and (2b) square zigzag lattice of silicon. 


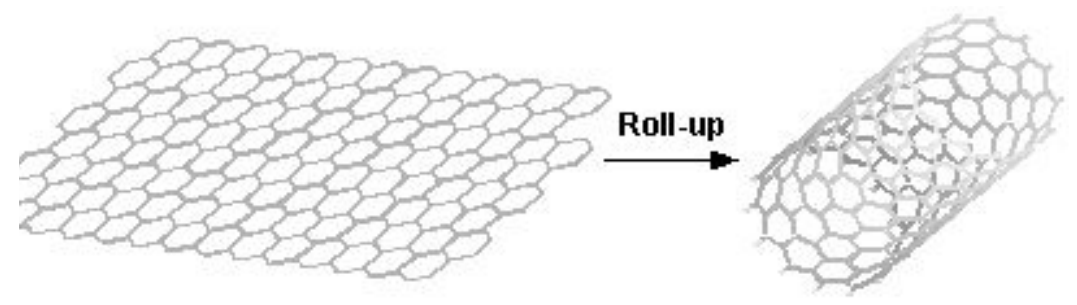

Fig 3: Rolling of hexagonal Si sheet to form SiNT.

\subsection{The Model Potential}

The interaction between silicon atoms on the same tube is modeled by an effective short range potential of Tersoff form [17]. The potential energy between the atoms $i$ and $j$ on the same tube separated by a distance $r_{i j}$ is of the form:

$$
\begin{aligned}
& V\left(r_{i j}\right)=f_{c}\left(r_{i j}\right)\left[f_{R}\left(r_{i j}\right)+b_{i j} f_{A}\left(r_{i j}\right)\right] \text {. where } \\
& f_{R}(r)=A e^{-\lambda_{1} r} ; f_{A}(r)=-B e^{-\lambda_{2} r} .
\end{aligned}
$$

After we generate the coordinates of Silicon atoms on a Si nanotube. The nearest and next neighbours of each atom are found and energy per atom is calculated using the potential in eq.1. The total energy of the tube is given by

$$
U=\sum_{i j} V\left(r_{i j}\right)
$$

The coordinates of each atom are modified in very small steps and energy is compared with previous energy. The modified coordinate is accepted if this energy is smaller than the previous one, otherwise we continue with the previous value of the coordinate. This is done successively for all the. coordinates and such a cycle is repeated several times till the energy of the tube is minimized. This is the process of relaxation under the given potential. Based on a high-resolution TEM micrograph of the obtained nanotubes, the authors suggested a multiwalled structure with an interlayer spacing of $0.31 \mathrm{~nm}$, covered with a thick oxide layer that can be removed by $\mathrm{HF}$ treatment.[12,13] The interlayer spacing is intriguing, since a larger spacing of about $0.4 \mathrm{~nm}$ is expected based on the Van der Waals radius of Si $(0.21 \mathrm{~nm})$. Using the scale bar of the TEM micrographs reported in the two reports,[12,13] Perepichka and Rosei [16] obtained interlayer spacings of 0.22 and $0.29 \mathrm{~nm}$, respectively, which are quite different from the claimed spacing of $0.31 \mathrm{~nm}$. For our calculations we used the mean value of 0.22 and $0.29 \mathrm{~nm}$ that is $0.255 \mathrm{~nm}$. 

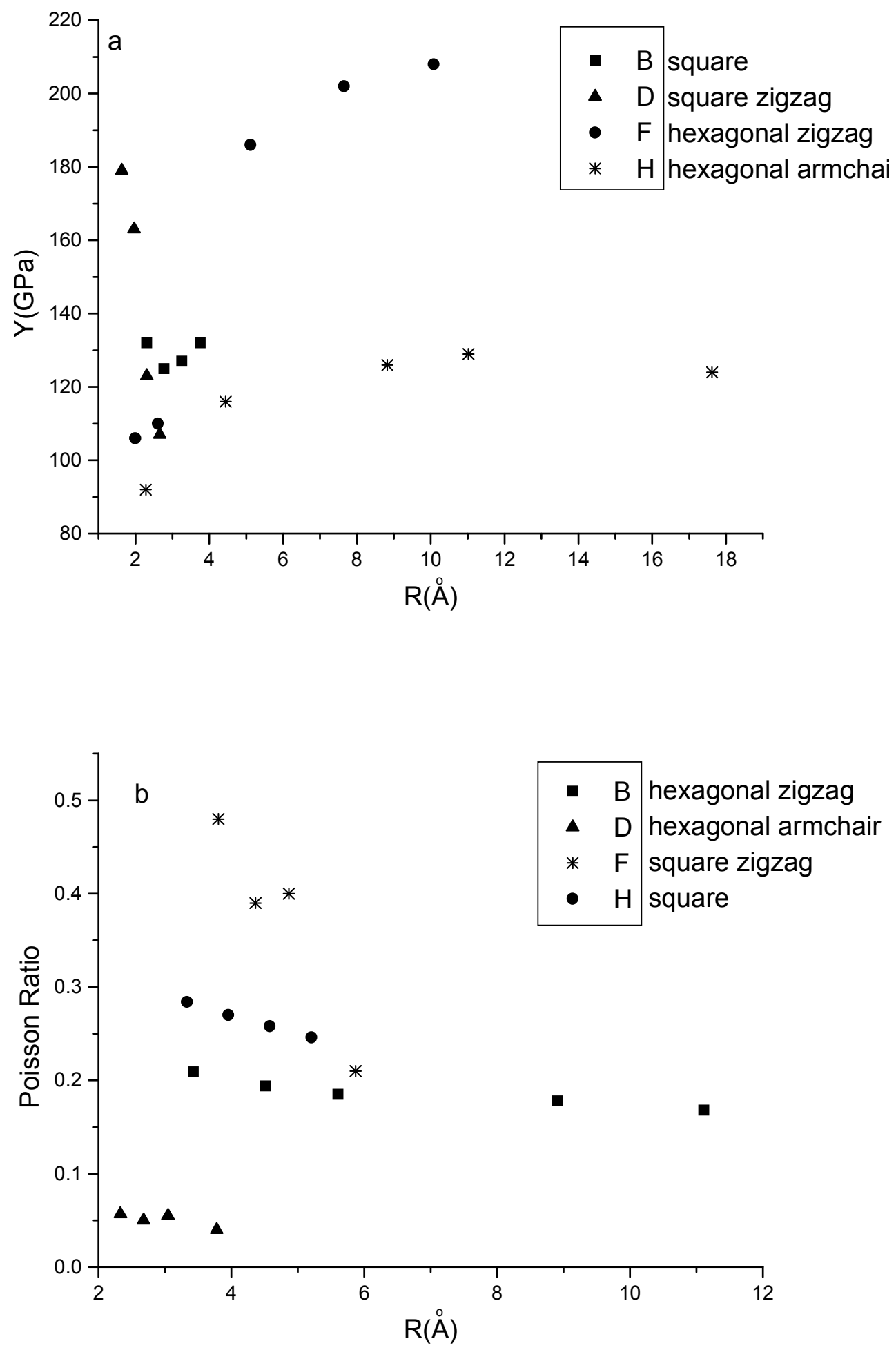


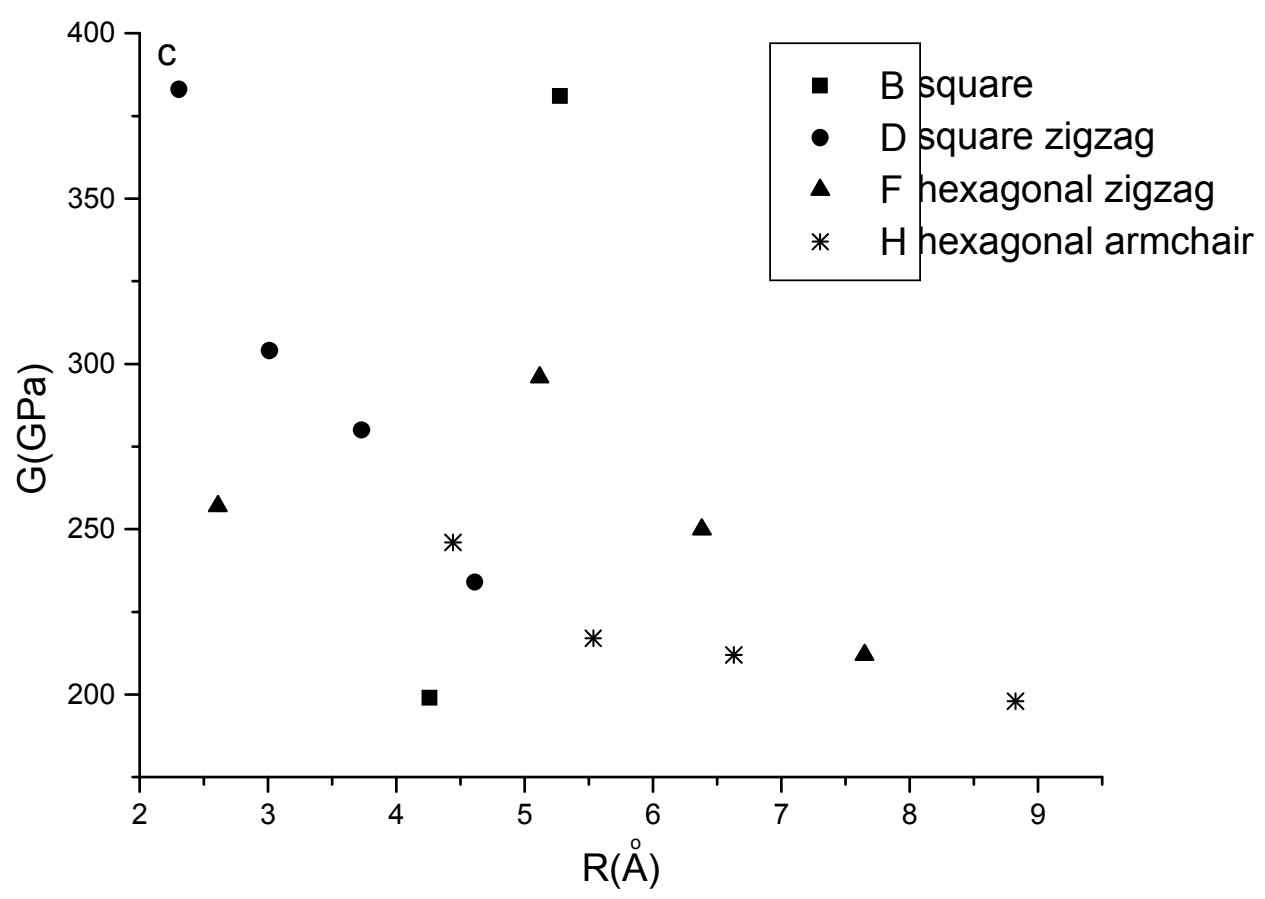

Fig 4 Variation of(a) Y, (b) Poisson ratio and (c) $G$ with radius for SiNTs

\subsection{Young's Modulus, Shear Modulus And Poisson Ratio Of sp2 And sp3 SiNT}

By applying the stress along the tube axis, Young modulus of SiNT has been calculated following the procedure used to compute $\mathrm{Y}$ values for CNTs and BNNTs [14,15] After getting the final position coordinates of silicon atoms on the surface of Si nanotube of given chirality $(\mathrm{n}, \mathrm{m})$, type (sp3/sp2) and length, we then consider the same tube under longitudinal stress. This stress is simulated by keeping the mutual distance between end rings in case of Si tube fixed at slightly larger (elongation) or smaller (compression) than normal. The coordinates of the rest of the atoms of the tube are varied with this constraint till minimum energy is obtained keeping the cylindrical shape of SiNT intact. Seifert et al. [4] calculated Young's modulus of 70-80 GPa for sp3 SiNTs over an order of magnitude lower than for carbon nanotubes. According to our calculations (Fig 4) Y values vary between $100 \mathrm{GPa}$ and $180 \mathrm{GPa}$ for sp3 SiNTs of smaller radii. For sp2 SiNTs the values vary slightly with radius and follow the same pattern as of CNTs [14] and the Y lies in the range of 100-200 GPa that is 10-20 times lower than CNTs. The effects of tube diameter and chirality are also investigated (fig 4). Our results show that like CNTs theYoung's modulus of small diameter $(n, n)$ and $(n, 0)$ SiNTs increases with nanotube diameter and reaches a constant value in the range of Gpa. But for square plane SiNTs Y falls with radius and for square zigzag SiNTs Y value shows a minima of $120 \mathrm{Gpa}$ at about $0.3 \mathrm{~nm}$ radius. Poisson ratio decreases as radius is increased for all the four types. For square SiNT the value is very low but for square zigzag it is more like rubber or lead. For conventional (n,n) and (n.0) SiNTs the value is about 0.2-0.3 which is more than both CNTs [14] and BNNTs [15] where it is in the range of 0.15 . The shear modulus decreases with radius of SiNT for all the four types of SiNTs. 


\section{Discussion and Conclusions:}

In this study on SiNTs using the well tested form of Tersof potential we have been able to find the values of Young's modulus, poisson ratio and shear modulus of a numberof SiNTs of various sizes and shapes. According to our calculations the existence of sp3 square and square zigzag SiNTs is validated. The values of Young's Modulus are slightly large than the reference given above. This may be due to the smaller value of inter layer thickness $(0.255 \mathrm{~nm})$ where as it is taken as $0.31 \mathrm{~nm}$ by some others. As is evident from values of poisson ratio SiNTs are expected to be more like metals except square SiNT. As SiNTs can find their vital applications due to their metallic and biodegradable nature indepth investigation is required to further our knowledge about nanostructures.

\section{References:}

[1] bbc.co.uk, BBC News Nature / Science, June 2004

[2] J. Bai, X.C.Zeng, H.Tanaka, J.Y.Zeng, Proc. Natl. Acad. Sci. USA, 101 (2004) 2664.

[3] S.B.Fagan, R.J.Baierle, R.Mota, A.J.R.da Silva, A.Fazzio, Phys.Rev.B 61 (2000) 9994.

[4] G.Seifert, Th.Kçhler, H.M.Urbassek, E.HernHndez, Th..Frauenheim, Phys. Rev. B 63, (2001) 193409.

[5] M Zhang, Y.H.Kan, Q.J.Zang, ZM.Su, R.S.Wang, Chem.Phys.Lett. 379 (2003) 81.

[6] J Hu, M.Ouyang, P.Yang, C.M.Lieber, Nature 399, (1999) 48.

[7].D.D.D.Ma, C.S.Lee, F.C.K.Au, S.Y.Tong, S.T.Lee, Science, 299, (2003) 1874.

[8] H Matsumoto, S.Kyushin, M.Unno, R.Tanaka, J.Organomet. Chem. 611 (2002) 52.

[9] J.Sha, J.Niu, X.Ma, J.Xu, X.Zhang, Q.Yang, D.Yang, Adv. Mater.14,.(2002) 1219.

[10] Schmidt and Eberl (O. G. Schmidt, K. Eberl, Nature, 410 (2001))168.

[11] S.Y.Jeong, J.Y.Kim, H.D.Yang, B.N.Yoon, S.H.Choi, H.K.Kang, C.W.Yang, Y.H. Lee, Adv. Mater. 15 (2003) 1172.

[12] Y.W. Chen, Y. H. Tang, L. Z. Pei, C. Guo, Adv. Mater. 17 (2005) 564.

[13] Y.H.Tang, L.Z.Pei, Y.W.Chen, C.Guo, Phys. Rev. Lett., 95 (2005) 116102.

[14] S.Gupta., KDharamvir, V.K.Jindal, Phy. Rev. B 72 (2005) 165428.

[15] Veena Verma, V K Jindal and Keya Dharamvir Nanotechnology 18 (2007) 435711 doi:10.1088/0957-4484/18/43/435711

[16] Dmitrii , F.Perepichka and Federico Rosei, Small 2, No.1 (2006) 22 - 25.

[17] J. Tersoff. Phys. Rev. B 37 (1988) 6991. 